\title{
Interpreting Discordant Monosomy 3 FISH and Chromosomal Microarray Analysis Results in Uveal Melanoma
}

Nicholas Coley

University of California San Diego School of Medicine

Christopher Long

University of California San Diego School of Medicine

Simrin Sennik

Memorial University of Newfoundland

John Thorson

University of California San Diego Health System

Jonathan Lin ( $\boldsymbol{D}$ jlinn@stanford.edu )

University of California, San Diego Department of Pathology https://orcid.org/0000-0002-7438-6941

\section{Case Report}

Keywords: uveal melanoma, monosomy 3, microarray, FISH

Posted Date: February 17th, 2020

DOI: https://doi.org/10.21203/rs.2.23660/v1

License: (c) (1) This work is licensed under a Creative Commons Attribution 4.0 International License. Read Full License 


\section{Abstract}

Background: Uveal melanoma is the most common primary ocular tumor in adults and causes morbidity through lymphovascular metastasis. The presence of monosomy 3 in uveal melanomas is one of the most important prognostic indicators for metastasis. Two major molecular pathology testing modalities to assess monosomy 3 are fluorescent in situ hybridization (FISH) and chromosomal microarray analysis (CMA). Here, we report two cases of discordant monosomy 3 test results in uveal melanoma enucleation specimens using these molecular pathology tests.

Case presentation: The first case is a uveal melanoma from a 51-year-old male that showed no evidence of monosomy 3 by CMA, but was subsequently detected by FISH. The second case is a uveal melanoma from a 49-year-old male that showed monosomy 3 at the limit of detection by CMA that was not detected by subsequent FISH.

Conclusions: These two cases underscore the differences of each testing modality for monosomy 3 . The high percentage of cells with one chromosome 3 signal requisite for a positive monosomy 3 FISH result may not be sensitive enough to detect a low level of monosomy 3 that CMA can detect. Conversely, a small uveal melanoma with monosomy 3 may be missed by CMA owing to background DNA from cytologically normal retina and other ocular tissues. Our cases suggest that both testing methods should be pursued for uveal melanoma, with a single positive result for either test interpreted as presence of monosomy 3.

\section{Background}

Uveal melanoma has an incidence of 5.2 per million people and is the most common primary ocular tumor in adults [1]. Local recurrence is rare following identification and treatment, but up to $50 \%$ of patients develop metastatic disease, most commonly to the liver and lungs [2]. There are no treatments for metastatic disease with poor 5-year survival outcomes [1]. Thus, the discovery of prognostic indicators and predictors of development of metastatic disease has been an important area of research.

There are a number of clinical, histopathologic, and cytogenetic features that have been associated with an increase in metastatic risk. From a clinical perspective, age, gender, tumor size, tumor location, and presence of extraocular extension have been shown to have significant prognostic value [3]. Histopathologically, there are a number of factors that convey prognostic value including tumor cell morphology (e.g., epithelioid vs spindle) and mitotic activity, as well as various receptor and antigen expression levels [4]. Some of the most significant prognostic indicators for uveal melanoma, however, are its cytogenetic features.

Chromosomal abnormalities, such as chromosome 3 loss, have been shown to be predictive of diseasespecific mortality [5]. Monosomy 3 has a reported frequency of approximately $40 \%$ in uveal melanomas, making it one of the most common genetic alterations in this disease [6]. Originally established as a significant prognostic indicator in 1996, chromosome 3 loss is one of the most well established predictive 
factors in uveal melanoma [7]. In the original study, Prescher et al., examined 54 cases of uveal melanoma treated by enucleation. Monosomy 3 was detected via karyotyping and comparative genomic hybridization in 30 of these cases. On 3-year follow up, $50 \%$ of these cases had developed metastases, while no patients in the disomy 3 group had developed metastases. Since 1996, additional studies have confirmed the negative prognostic value of monosomy 3 and suggest its value is most useful when interpreted in the context of clinical and pathology features such as tumor size and cell type of the tumor $[5,8]$. Current research has focused on partial chromosomal alterations. The frequency of these alterations varies widely in the literature and their clinical significance is still under debate. Some reports have implicated these alterations in metastatic disease, while other results have suggested they have no significant clinical value [9].

Two of the most common molecular pathology techniques used to assess for monosomy 3 are FISH and CMA. FISH uses fluorochromes linked to DNA probes to rapidly identity and visualize targeted DNA sequences under the fluorescence microscope. First used in uveal melanoma in 1997, FISH is now widely used in the study of uveal melanoma cytogenetics, specifically in the detection of monosomy 3 [10].

Another common technique is $\mathrm{CMA}$, which provides an alternative method for assessing chromosome copy number variations. One form of CMA, known as array comparative genomic hybridization (CGH), combines the use of a microarray with the classic $\mathrm{CGH}$ technique of in which two fluorochromes are used to differentially label genomic material from two samples, a test and a control. The labelled samples are then competitively hybridized to either reference metaphase spreads (classical CGH) or a microarray created from cloned DNA fragments which represent the normal genome (array CGH). Signal intensity is then compared between test and control fluorochromes to identify variations in copy numbers in the test genome [11]. CGH was first described in the analysis of chromosomal aberrations in uveal melanoma in 1996 [12]. Overtime, CGH has been recognized as an effective method for detection of chromosome 3 abnormalities and is especially useful for localization of small chromosomal aberrations to specific chromosomal regions [13]. Additionally, CGH has been demonstrated to effectively identify genomic copy variants that can distinguish benign nevi from melanomatous lesions [14]. An alternative form of CMA, single nucleotide polymorphism (SNP)-based chromosomal microarrays, uses the absolute intensity of a single fluorescent signal at a polymorphic chromosomal position to detect a gain or loss of genomic material at that chromosomal location [15]. Both SNP-based chromosomal microarray and FISH were implemented for both cases in this study.

Although widely used in practice today, these methods each have a number of limitations.

Based on a review published in 2012, the mean detection rate of monosomy 3 using various FISH techniques studied was only $48 \%$ [16]. Reliability is also influenced by tissue sampling technique, with highest rates of detection of monosomy 3 associated with fresh tissues when compared to fine needle aspiration biopsy [17]. Perhaps the most significant factor contributing to the limited success of the FISH technique is tumor heterogeneity seen in uveal melanoma. When FISH is used to detect chromosomal 3 aberrations in fine needle aspirations, it is possible the sampled area will not contain enough cells with 
chromosomal 3 loss to meet the threshold for detection [18]. Thus, FISH results are heavily influenced by both sample area and threshold levels $[19,20]$. An additional limitation of the FISH technique is its limited ability to detect partial chromosome loss. For example, centromeric probes may be effective at detecting monosomy 3, but often miss partial deletions and thus underestimate chromosomal loss [21].

CMA also has limitations in its ability to detect chromosome 3 aberrations. Similar to FISH, CMA detection of monosomy 3 in uveal melanoma is limited by tumor heterozygosity as well overall malignant cell content. As discussed above, uveal melanomas exhibit tumor heterozygosity with respect to chromosome 3 copy number. Thus, CMA results are significantly influenced by detection thresholds used during analysis. Previous research has demonstrated cases in which CMA has miss-reported chromosome 3 status using a conventional cutoff of $20 \%$ of cells for detection [22]. Lastly, sample type and preparation have also been shown to affect CMA results. Previous research has demonstrated a significant amount of background signal present in material obtained from samples embedded in paraffin which can affect the ability of CMA to detect chromosomal aberrations in these samples [23].

\section{Case Presentation}

Patient \#1 is a 51 -year-old male who presented to ophthalmology for progressive left-sided vision loss. Ophthalmoscopic examination revealed a mushroom-shaped pigmented lesion emanating from the posterior uvea overlying the optic nerve, proven to be melanoma by fine needle aspiration (FNA) biopsy. He underwent a 7-day surgical placement of a radioiodine plaque overlying the tumor 1 month after initial pathologic diagnosis. A clinically detected local recurrence at the periphery of the plaque site 60 months after pathologic diagnosis underwent multiple rounds of laser ablation. He ultimately underwent enucleation 79 months after pathologic diagnosis. His most recent imaging 92 months after initial pathologic diagnosis demonstrates numerous liver metastasis and local left orbital tumor extension tracking along the optic nerve to involve the optic chiasm.

Patient \#2 is a 49-year-old male who presented to ophthalmology for an enlarging pigmented mass present on his right iris. He underwent surgical radioiodine plaque placement (removed after 7 days) at the time of FNA biopsy that demonstrated uveal melanoma. He underwent a 7-month trial of sunitinib shortly after initial pathologic diagnosis. 15 months after pathologic diagnosis magnetic resonance imaging (MRI) abdomen demonstrated two biopsy-proven metastatic liver lesions, subsequently treated with radioablation. He underwent enucleation 36 months after pathologic diagnosis and a right apical lung lesion biopsied at 38 months demonstrated metastatic melanoma. An enlarging right apical lung lesion is the only metastatic disease detected by computed tomography (CT) chest in his most recent imaging scan performed 48 months after pathologic diagnosis.

\section{Materials And Methods}

Oncoscan microarray was performed at the University of California San Diego (UCSD) Center for Advanced Laboratory Medicine (CALM) using the Affymetrix/Thermofisher OncoScan platform (catalog 
number: 902695) on DNA extracted from sections of formalin-fixed, paraffin-embedded (FFPE) tissue. Monosomy $3 \mathrm{FISH}$ was performed at Mayo Clinic Laboratories (test ID: UMM3F), utilizing the D3Z1 centromeric probe (Abbot Molecular) and the BCL6 long arm probe (Mayo Laboratories). Greater than $28 \%$ of 200 cells lacking two chromosome 3 signatures is requisite for a positive monosomy 3 result using this assay. Next generation sequencing (NGS) was performed at the UCSD CALM on DNA extracted from FFPE tissue using a laboratory developed 397 gene hybrid capture-based assay with analysis on an Illumina HiSeq 2500 instrument (Illumina, San Diego, CA).

The images of FFPE, hematoxylin and eosin (H\&E) stained whole eye cross sections were taken using the Aperio AT2 whole slide scanner. The remaining images were captured using the Olympus SC30 camera with cellSens software attached to an Olympus BX43 microscope.

The UCSD institutional review board approved the use of the collected tissue samples and associated clinical information for this study. The research was Health Insurance Portability and Accountability Act (HIPAA) compliant and adhered to the principles of the Declaration of Helsinki.

\section{Results}

The uveal melanoma from patient \#1 arises from the posterior uvea overlying the optic nerve (figure 1A). Some scleral invasion is present near the optic nerve, but no extrascleral extension is present. This tumor assumes a primarily epithelioid morphology with variable pigmentation (figure 1B). While OncoScan microarray did not detect monosomy 3, FISH did detect evidence of monosomy 3 in $63.5 \%$ of cells counted (positive cutoff $>28 \%$ of 200 cells) (table 1 , supplementary figure $1 \mathrm{~A}$ ). No clinically significant mutations were detected by the next generation sequencing (NGS) panel (table 1).

The uveal melanoma from patient \#2 arises from the ciliary body (Figures 1C). This tumor assumes a primarily spindle cell morphology (figure 1D). Necrotic debris and numerous melanophages are scattered throughout this tumor secondary to radioiodine plaque placement (figure 1D insert). While the tumor did not meet criteria for monosomy 3 by FISH, OncoScan microarray detected monosomy 3 at the limit of detection (table 1, supplementary figure 1B). No clinically significant mutations were detected by the NGS panel for either tumor (table 1).

\section{Discussion}

These two cases highlight the diagnostic strengths and pitfalls of CMA and FISH for assessing monosomy 3 status in uveal melanoma. CMA utilizes DNA extracted from an entire tumor sample and inevitably includes some background or interspersed normal tissues. Consequently, CMA may fail to detect monosomy 3 in a small subclone or the DNA from normal tissue may obfuscate low-level loss of chromosome 3 within a tumor. The detection of monosomy 3 by FISH not detected by CMA in patient \# 1 may reflect this phenomenon. FISH, however, samples a narrow plane of tissue and requires a relatively high proportion of cells with a single probe signal to minimize false positive results. Thus, FISH may miss 
monosomy 3 at a low-level that CMA can routinely detect. The detection of monosomy 3 by CMA not detected by FISH in patient \#2 may reflect this phenomenon. Overall, these two cases highlight the strengths and weaknesses of FISH and CMA as assays to detect monosomy 3 in uveal melanoma. We suggest that both FISH and CMA should be performed for uveal melanoma, with a positive result for either test interpreted as presence of monosomy 3.

\section{Abbreviations}

CALM: Center for Applied Laboratory Medicine

CGH: comparative genomic hybridization

CMA: chromosomal microarray analysis

CT: computed tomography

FFPE: formalin-fixed, paraffin-embedded

FISH: fluorescent in situ hybridization

HIPAA: Health Insurance Portability and Accountability Act

MRI: magnetic resonance imaging

NGS: next generation sequencing

SNP: single nucleotide polymorphism

UCSD: University of California, San Diego

\section{Declarations}

Ethics approval and consent to participate: Tissue derived from both patients in this retrospective study was consented for potential research use under the UC San Diego Health System consent for treatment forms.

Consent for publication: No personal identification from either patient is presented in this manuscript per HIPAA regulations.

Availability of data and materials: FFPE tissue from either case can be obtained for additional studies following approval of a material transfer agreement. Microarray data from each case can be provided deidentified of HIPAA sensitive information upon request as well as the original FISH reports from Mayo laboratories.

Competing interests: There are no competing interests or private financial interests to disclose.

Funding: Coverage for molecular testing (FISH and microarray) was provided by each patient's health care insurer (Cigna for case \#1 and Blue Cross for case \#2) and the UC San Diego Health Care System.

Authors' contributions: NC interpreted the initial molecular tests, took all images, and is responsible for authoring the majority of the manuscript. CL wrote the majority of the background sections with 
additional contributions by SS. JL and JT reviewed the finished manuscript and served as final editors/mentors.

Acknowledgements: We thank the staff at the UC San Diego Center for Applied Laboratory Medicine (CALM) for performing all microarray testing. We thank Mayo Laboratories in Rochester MN for performing all FISH testing.

\section{References}

1. Aronow ME, Topham AK, Singh AD. Uveal Melanoma: 5-Year Update on Incidence, Treatment, and Survival (SEER 1973-2013). Ocul Oncol Pathol, 2018, 4:145-51.

2. Carvajal RD, Schwartz GK, Tezel T, Marr B, Francis JH, Nathan PD. Metastatic disease from uveal melanoma: treatment options and future prospects. Br J Ophthalmol, 2017, 101:38-44.

3. Walter SD, Chao DL, Feuer W, Schiffman J, Char DH, Harbour JW. Prognostic Implications of Tumor Diameter in Association With Gene Expression Profile for Uveal Melanoma. JAMA Ophthalmol, 2016, 134:734.

4. Kaliki S, Shields C, Shields J. Uveal melanoma: Estimating prognosis. Indian J Ophthalmol, 2015, 63:93.

5. Damato B, Duke C, Coupland SE, Hiscott P, Smith PA, Campbell I, et al. Cytogenetics of uveal melanoma: a 7-year clinical experience. Ophthalmology, 2007, 114:1925-31.

6. Prescher G, Bornfeld N, Becher R. Nonrandom Chromosomal Abnormalities in Primary Uveal Melanoma. JNCl J Natl Cancer Inst, 1990, 82:1765-9.

7. Prescher G, Bornfeld N, Hirche H, Horsthemke B, Jöckel KH, Becher R. Prognostic implications of monosomy 3 in uveal melanoma. Lancet (London, England), 1996, 347:1222-5.

8. Scholes AGM, Damato BE, Nunn J, Hiscott P, Grierson I, Field JK. Monosomy 3 in uveal melanoma: correlation with clinical and histologic predictors of survival. Invest Ophthalmol Vis Sci, 2003, 44:1008-11.

9. Abdel-Rahman MH, Christopher BN, Faramawi MF, Said-Ahmed K, Cole C, McFaddin A, et al. Frequency, molecular pathology and potential clinical significance of partial chromosome 3 aberrations in uveal melanoma. Mod Pathol, 2011, 24:954-62.

10. Sisley K, Rennie IG, Parsons MA, Jacques R, Hammond DW, Bell SM, et al. Abnormalities of chromosomes 3 and 8 in posterior uveal melanoma correlate with prognosis. Genes Chromosomes Cancer, 1997, 19:22-8.

11. Theisen A. Microarray-based comparative genomic hybridization (aCGH). Nat Educ, 2008, 1:45.

12. Speicher MR, Prescher G, du Manoir S, Jauch A, Horsthemke B, Bornfeld N, et al. Chromosomal gains and losses in uveal melanomas detected by comparative genomic hybridization. Cancer Res, 1994, 54:3817-23. 
13. Naus NC, van Drunen E, de Klein A, Luyten GP, Paridaens DA, Alers JC, et al. Characterization of complex chromosomal abnormalities in uveal melanoma by fluorescence in situ hybridization, spectral karyotyping, and comparative genomic hybridization. Genes Chromosomes Cancer, 2001, 30:267-73.

14. Mesbah Ardakani N, Thomas C, Robinson C, Mina K, Harvey NT, Amanuel B, et al. Detection of copy number variations in melanocytic lesions utilising array based comparative genomic hybridisation. Pathology, 2017, 49:285-91.

15. Coughlin $\mathrm{CR}$, Scharer GH, Shaikh TH. Clinical impact of copy number variation analysis using highresolution microarray technologies: advantages, limitations, and concerns. Genome Med, 2012, 4:80.

16. Aronow M, Sun Y, Saunthararajah Y, Biscotti C, Tubbs R, Triozzi P, et al. Monosomy 3 by FISH in Uveal Melanoma: Variability in Techniques and Results. Surv Ophthalmol, 2012, 57:463-73.

17. Naus NC, Verhoeven ACA, van Drunen E, Slater R, Mooy CM, Paridaens DA, et al. Detection of genetic prognostic markers in uveal melanoma biopsies using fluorescence in situ hybridization. Clin Cancer Res, 2002, 8:534-9.

18. Maat W, Jordanova ES, van Zelderen-Bhola SL, Barthen ER, Wessels HW, Schalij-Delfos NE, et al. The heterogeneous distribution of monosomy 3 in uveal melanomas: implications for prognostication based on fine-needle aspiration biopsies. Arch Pathol Lab Med, 2007, 131:91-6.

19. Schoenfield L, Pettay J, Tubbs RR, Singh AD. Variation of monosomy 3 status within uveal melanoma. Arch Pathol Lab Med, 2009, 133:1219-22.

20. Bronkhorst IHG, Maat W, Jordanova ES, Kroes WGM, Schalij-Delfos NE, Luyten GPM, et al. Effect of heterogeneous distribution of monosomy 3 on prognosis in uveal melanoma. Arch Pathol Lab Med, 2011, 135:1042-7.

21. Damato B, Coupland SE. Translating uveal melanoma cytogenetics into clinical care. Arch Ophthalmol (Chicago, III 1960), 2009, 127:423-9.

22. White JS, McLean IW, Becker RL, Director-Myska AE, Nath J. Correlation of comparative genomic hybridization results of 100 archival uveal melanomas with patient survival. Cancer Genet Cytogenet, 2006, 170:29-39.

23. Mensink HW, Kiliç E, Vaarwater J, Douben H, Paridaens D, de Klein A. Molecular cytogenetic analysis of archival uveal melanoma with known clinical outcome. Cancer Genet Cytogenet, 2008, 181:10811.

\section{Table}

Table 1. No clinically significant mutations were detected by NGS for either tumor. The uveal melanoma from patient \#1 showed focal gain of chromosome 9 and no detectable chromosomal losses. However, this tumor demonstrated monosomy 3 by FISH analysis. The uveal melanoma from patient \#2 showed several chromosomal gains in addition to monosomy 3 at the limit of detection. FISH analysis did not detect monosomy 3 for this tumor. 


\begin{tabular}{|c|c|c|}
\cline { 2 - 3 } \multicolumn{1}{c|}{} & Patient \#1 & Patient \#2 \\
\hline$\underline{\text { Mutations }}$ & No clinically significant variants detected. & $\begin{array}{c}\text { No clinically significant variants } \\
\text { detected. }\end{array}$ \\
\hline$\underline{\text { Chromosomal }}$ & $\begin{array}{c}2.27 \text { Mb gain in 9q33.1 encompassing 6 genes } \\
\text { (ASTN2, SNORA70C, LOC101928797, TLR4, } \\
\text { LINC02578, BRINP1). }\end{array}$ & $\begin{array}{c}\text { Gain of chromosomes 7, 8, 18, 19, } \\
\text { and 22. }\end{array}$ \\
\hline$\underline{\text { Chromosomal }}$ & Nosses chromosomal losses detected. & $\begin{array}{c}\text { Monosomy 3 at limit of detection } \\
\text { with additional losses of } \\
\text { chromosomes 4, 12, and 16q. }\end{array}$ \\
\hline$\underline{\underline{\text { Monosomy 3 }}}$ & Positive, 63.5\% of 200 cells counted (28\% \\
cutoff). & Negative. \\
\hline
\end{tabular}

\section{Figures}
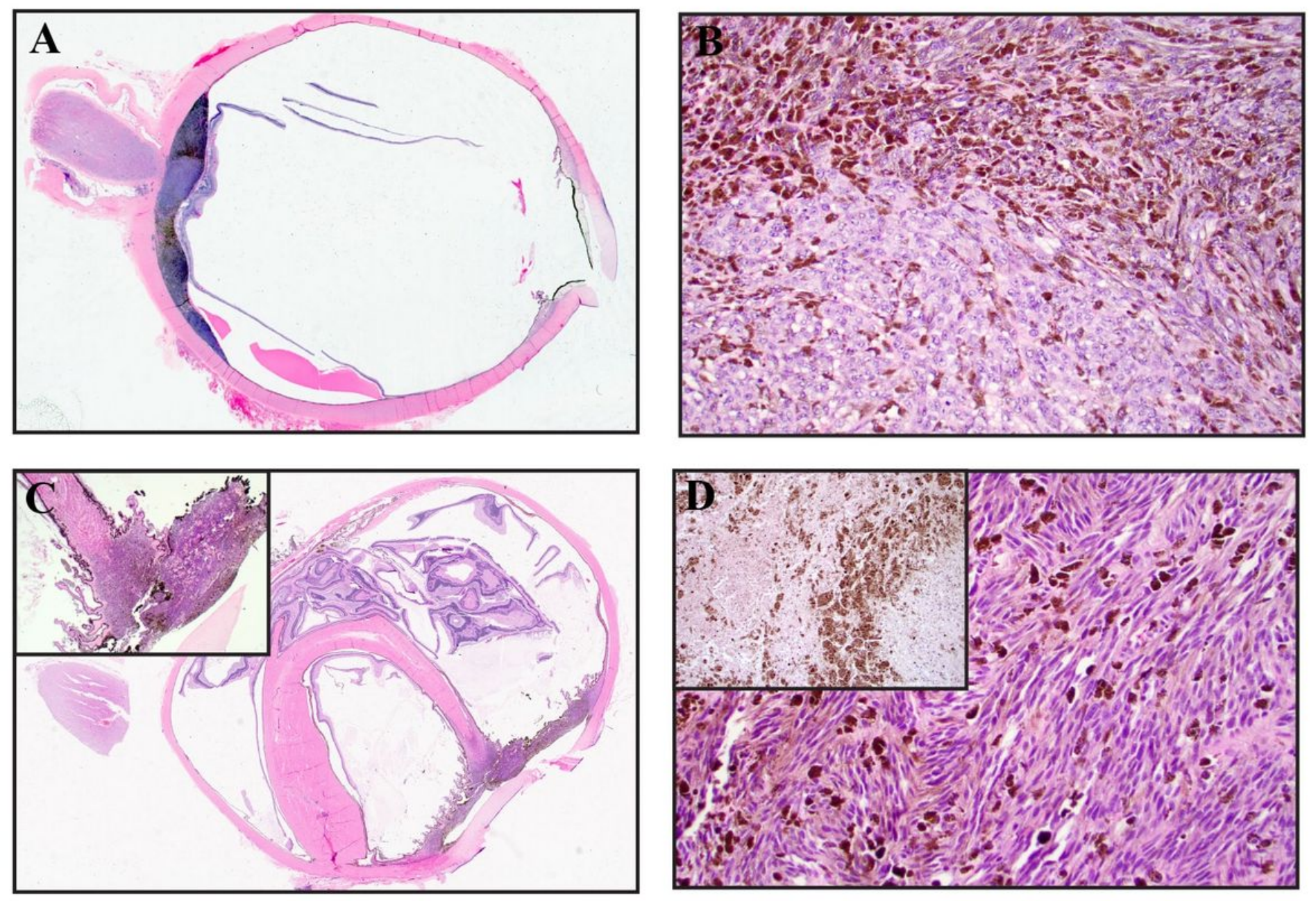
The melanoma from patient \#1 arises from the posterior uvea overlying the optic nerve (A). A foci of scleral invasion adjacent to the optic nerve is present, but no extrascleral extension is noted. The tumor assumes a primarily epithelioid morphology (B). The melanoma from patient \#2 arises from the ciliary body (C). The tumor assumes a primarily spindle cell morphology (D). The tumor from patient \#2 showed extensive necrosis and presence of melanophages (insert $\mathrm{D}$ ) secondary to radioiodine plaque treatment.

\section{Supplementary Files}

This is a list of supplementary files associated with this preprint. Click to download.

- Monosomy3paperSupplementallmage01.pdf 